\title{
Development and characterization of curcumin-loaded polycaprolactone nanoparticles by Design of Experiments
}

\author{
Andreea Lavinia TINCA ${ }^{1}$, Sonia IURIAN¹, Lucia Maria RUS², Alina PORFIRE ${ }^{1}$, \\ Lucia Ruxandra TEFAS ${ }^{1}$ \\ ${ }^{1}$ Department of Pharmaceutical Technology and Biopharmacy, Faculty of Pharmacy, \\ "Iuliu Hatieganu" University of Medicine and Pharmacy, Cluj-Napoca, Romania \\ ${ }^{2}$ Department of Pharmaceutical Analysis, Faculty of Pharmacy, \\ "Iuliu Hatieganu" University of Medicine and Pharmacy, Cluj-Napoca, Romania
}

\begin{abstract}
Objectives. Design of Experiments (DOE) is a powerful tool for identifying important formulation factors and process parameters in drug design and manufacturing, to ensure certain product specifications. This study aimed at manufacturing curcumin-loaded polycaprolactone nanoparticles (CURC-PCL NP), and used DOE in order to understand how formulation and process variables impact the drug delivery system's characteristics. Polymeric nanoparticles are a suitable approach for incorporating hydrophobic drugs such as curcumin, in order to maximize the numerous beneficial effects, and to minimize the side effects and shortcomings such as low water-solubility and bioavailability.

Material and methods. The CURC-PCL NP were prepared by the solvent displacement method. The effects of various formulation and process variables on the nanoparticles' characteristics were investigated by employing a fractional factorial design with 19 runs.

Outcomes. Regression equations were generated to predict the impact of changing variables on particle size, size distribution, zeta potential, drug content and entrapment efficiency. The statistical analysis indicated that the chosen model was robust, reliable and assured good experimental control.

Conclusions. Overall, this study shows that DOE is useful in achieving the desirable goals in polymeric nanoparticle development, in the shortest time possible and with a reduced number of experiments.
\end{abstract}

Keywords: curcumin, polycaprolactone, polymeric nanoparticles, Design of Experiments

\section{INTRODUCTION}

Curcumin (CURC) or

diferuloylmethane is a natural yellow compound extracted from the root of Curcuma longa, also known as turmeric, from the Zingiberaceae family of plants [1]. Is possesses a symmetric molecule containing three main chemical entities: two aryl moieties substituted with ortho-methoxy phenolic groups which are linked together by a seven-carbon atom chain containing an $\alpha$-, $\beta$-unsaturated $\beta$-diketone moiety [2]. CURC is the major compound of turmeric $(60-70 \%)$, but other two structurally related compounds, namely demethoxycurcumin (20-30\%) and bisdemethoxycurcumin (3-10\%), are also present but in lower amounts [2,3]. For many centuries, CURC has been used as a spice and 
supplement in traditional Asian cuisine and medicine, for its pleiotropic effects [4]. This polyphenol possesses significant antioxidant properties mainly through the phenolic and methoxy groups on the benzene rings and $\beta$-diketone moiety. Its ability to scavenge various reactive oxygen species and free radicals explains its beneficial effects in diseases associated with oxidative stress [1]. CURC has been reported to display anti-inflammatory, anticarcinogenic and antimicrobial properties, as well as protective effects towards a wide range of diseases, including cardiovascular and neurologic diseases, and diabetes [3,5]. Toxicity studies performed in animals revealed that CURC was well tolerated at high doses, without any toxic effects. Data from safety evaluation studies have led the US Food and Drug Administration (FDA) to classify it as "generally recognized as safe" (GRAS). In a recently published assessment report on Curcuma longa L. rhizome, the European Medicines Agency (EMA) reported that no serious side effects have been detected up to now in humans, therefore CURC can be regarded as safe [6]. However, its lipophilic nature, low solubility in water, extensive metabolism, and physicochemical instability compromise CURC's bioavailability, which hence limits its potential medical applications, and has led to its classification as a PAINS (panassay interference compounds) and IMP (invalid metabolic panaceas) candidate [1].

To circumvent these shortcomings, various strategies have been employed to improve the physicochemical and biopharmaceutical properties of CURC, such as its encapsulation in different nanocarrier-based drug delivery systems, including liposomes, polymeric micelles, cyclodextrin inclusion complexes, polymeric nanoparticles and solid lipid nanoparticles [5,7]. Among these types of systems, polymeric nanoparticles are a good option for encapsulating various drugs with low water solubility such as CURC, because of their advantageous characteristics in terms of ease of production, good biocompatibility, the wide range of polymeric matrices that can be used for their formulation, and impressive bio-imitative ability [8]. Polymeric nanoparticles have several advantages over other nanocarriers, such as improved stability compared to liposomes, therefore being able to protect the encapsulated drug to a higher extent. Also, they can exhibit a controlled drug release and prolonged blood circulation, and their properties can be tuned to meet specific delivery needs [1]. Biodegradable and biocompatible polymers are extensively employed for drug delivery systems because they can break down in the physiological environment and thus possess lower toxicity $[4,9]$.

Polycaprolactone $(\mathrm{PCL})$ is a promising choice for developing nanocarrier-based systems because of its favorable characteristics such as biodegradability, reduced degradation rate, high permeability to many drugs, and low toxicity $[4,10]$. PCL is a synthetic polyester obtained from petroleum products either by ring-opening polymerization of $\varepsilon$-caprolactone with various cationic, anionic or co-ordination catalysts, or by free radical ring-opening polymerization of 2-methylene-1-3-dioxepane [11]. In physiological conditions, $\mathrm{PCL}$ is slowly degraded by hydrolysis of 
the ester bond, and the resulting polymer fragments are completely metabolized and subsequently excreted after degradation [12]. $P C L$ is FDA approved for use in various medical applications [13]. This makes PCL a suitable and safe material for long-term use of nanoplatforms. Furthermore, PCL shows increased stability in vivo, good cellular uptake and controlled release of the payload [9]. Due to its increased hydrophobic nature, PCL seems an appropriate candidate for the encapsulation of lipophilic drugs like CURC.

A wide range of preparation methods are available for polymeric nanoparticle manufacturing. The solvent displacement method also referred to as the nanoprecipitation method, is recommended for the encapsulation of lipophilic drugs in polymeric nanoparticles. However, a major drawback of this method is the formation of large aggregates as a by-product in the polymer precipitation process $[14,15]$. In order to control the grown of the nanoparticles and stabilize them, different polymeric surfactants or stabilizers are used. Nonionic surfactants such as Tween 80 , and polymeric stabilizers like polyvinyl alcohol (PVA) and Pluronic polymers are frequently employed in PCL nanoparticle production [16].

In the development of nanoformulations, there are generally complex relationships between the input variables and the outcome. Understanding these correlations would require performing a great number of experiments, with all the possible combinations, which is undoubtedly laborious, timeconsuming and expensive [17]. Design of Experiments (DOE) is a powerful and useful tool in drug design and manufacturing which enables, with a minimum number of experiments, to explore these relationships and offers statistically valid interpretations of individual and interactive effects of the input parameters on the responses $[18,19]$. Different types of designs can be employed in nanoformulation development, and among these fractional factorial designs allow the investigation of a large number of parameters, through fewer experiments, while still providing the necessary information. However, since this type of DOE is made up of some of the possible combinations, interactions between factors cannot be explored [19].

The objective of this study was to formulate, prepare and characterize a PCL-based nanoparticulate system loaded with the natural polyphenol CURC. The influence of different formulation and process parameters on the properties of the PCL nanoparticles was investigated using DOE principles.

\section{MATERIAL AND METHODS}

\section{Material}

CURC, PCL (average MW 45000), and PVA (average MW 3000070000) were purchased from Sigma-Aldrich (St. Louis, MO, USA). Kolliphor P407 (Poloxamer 407, P407) was purchased from BASF SE (Ludwigshafen, Germany). Acetone was obtained from SC Chimreactiv SRL (Bucharest, Romania). Ethanol was obtained from Chemical Company SA (Iași, Romania). Acetonitril was purchased from Merck \& Co (Kenilworth, NJ, USA). All other reagents were of HPLC grade. 


\section{Methods}

\section{Differential scanning calorimetry (DSC)}

The DSC measurements were carried out on a Mettler Toledo DSC822 ${ }^{\mathrm{e}}$ (Mettler Toledo, Columbus, $\mathrm{OH}$, USA) equipment, using indium in aluminum pans for the calibration step. Approximately $2 \mathrm{mg}$ of sample were introduced in $40 \mu \mathrm{L}$ aluminum pans, covered with a pierced lid and sealed using a sealing press. The heating rate was $10^{\circ} \mathrm{C} / \mathrm{min}$, over a temperature range of $20-400^{\circ} \mathrm{C}$. Nitrogen was purged through the system at a rate of $50 \mathrm{ml} / \mathrm{min}$. An empty $40 \mu \mathrm{l}$ aluminum pan served as reference. Thermograms were obtained for each individual substance, namely CURC, PCL, P407 and PVA, and 1:1 (w/w) physical mixtures of CURC with each excipient. Data were recorded and analyzed with the STAR ${ }^{\text {e }}$ SW 12.10 software.

\section{Nanoparticle preparation}

The CURC-loaded PCL nanoparticles (CURC-PCL NP) were obtained by the solvent displacement method as previously described, with slight modifications [20]. Briefly, exact amounts of PCL and CURC were dissolved in a known volume of acetone. Subsequently, the organic solution was added dropwise to an aqueous PVA or P407 solution of known concentration, using a syringe system equipped with a 21-gauge needle. The obtained dispersion was kept under magnetic stirring on a MultiStirrer 6 (VELP Scientifica, Usmate Velate (MB), Italy) at room temperature, for approximately $12 \mathrm{~h}$, in order to completely remove the organic solvent. The resulting nanoparticle suspension was centrifuged (Sigma Laborzentrifugen $\mathrm{GmbH}$, Osterode am Harz, Germany) at 5000 rpm for $15 \mathrm{~min}$ at $25^{\circ} \mathrm{C}$. The sediment was discarded, and the final volume was adjusted to $25 \mathrm{ml}$ with distilled water.

\section{DOE}

A 6-factor and 2-level Fractioned Factorial Design Resolution IV was implemented using the Modde 12.1 software (Sartorius Stedim Biotech, Göttingen, Germany) to determine the influence of different formulation factors and process parameters on the characteristics of CURC-PCL NP. The studied independent variables were the concentration of PCL ( $\% \mathrm{w} / \mathrm{V})$, the type and concentration of stabilizer (\% w/V), the CURC:PCL ratio (w/w), the volume of acetone $(\mathrm{ml})$ and the stirring speed (rpm). Of the abovementioned parameters, only the type of stabilizer was a qualitative variable, while the rest were quantitative factors. Each independent quantitative variable was varied on two levels, coded with -1 and +1 , corresponding to the lowest and highest value, respectively, as indicated in Table 1. For the qualitative independent variable, two types of polymeric stabilizers were investigated, namely PVA and P407. The dependent variables or responses were the particle size $\left(n m, Y_{1}\right)$, the polydispersity index $\left(\mathrm{PDI}, \mathrm{Y}_{2}\right)$, the zeta potential $\left(\mathrm{mV}, \mathrm{Y}_{3}\right)$, the CURC content $\left(\mu \mathrm{g} / \mathrm{ml}, \mathrm{Y}_{4}\right)$ and the entrapment efficiency $\left(\%, Y_{5}\right)$. A total of 19 experiments were carried out according to the outlined experimental design matrix in Table 2.

Particle size and size distribution The average particle size and particle size distribution, expressed as PDI, of the CURC-PCL NP were measured by Dynamic Light Scattering (DLS) using a Zetasizer Nano ZS (Malvern Panalytical, Malvern, UK). Each sample was diluted with distilled water in a 
TABLE 1. Independent variables and their levels of variation

\begin{tabular}{|l|c|c|c|c|c|}
\hline \multirow{2}{*}{ Independent variable } & \multirow{2}{*}{ Symbol } & \multirow{2}{*}{ Type of variable } & \multicolumn{3}{c|}{ Level of variation } \\
\cline { 4 - 6 } & & & $-\mathbf{1}$ & $\mathbf{0}$ & $\mathbf{+ 1}$ \\
\hline PCL concentration (\% w/V) & $\mathrm{X}_{1}$ & Quantitative & 0.1 & 0.2 & 0.3 \\
\hline Stabilizer concentration (\% w/V) & $\mathrm{X}_{2}$ & Quantitative & 0.5 & 1 & 1.5 \\
\hline Stabilizer type & $\mathrm{X}_{3}$ & Qualitative & \multicolumn{2}{|c|}{ PVA } & \multicolumn{1}{|c|}{ P407 } \\
\hline CURC:PCL ratio (w/w) & $\mathrm{X}_{4}$ & Quantitative & 0.1 & 0.3 & 0.5 \\
\hline Volume of acetone (ml) & $\mathrm{X}_{5}$ & Quantitative & 5 & 7.5 & 10 \\
\hline Stirring speed (rpm) & $\mathrm{X}_{6}$ & Quantitative & 170 & 255 & 340 \\
\hline
\end{tabular}

TABLE 2. Experimental design matrix

\begin{tabular}{|c|c|c|c|c|c|c|c|c|}
\hline No. & Exp. No. & Run order & $\mathbf{X}_{1}$ & $\mathbf{X}_{2}$ & $\mathbf{X}_{3}$ & $\mathbf{X}_{4}$ & $\mathbf{X}_{5}$ & $\mathbf{X}_{6}$ \\
\hline 1 & N1 & 19 & -1 & -1 & PVA & -1 & -1 & -1 \\
\hline 2 & N2 & 10 & 1 & -1 & PVA & -1 & 1 & -1 \\
\hline 3 & N3 & 3 & -1 & 1 & PVA & -1 & 1 & 1 \\
\hline 4 & N4 & 14 & 1 & 1 & PVA & -1 & -1 & 1 \\
\hline 5 & N5 & 7 & -1 & -1 & P407 & -1 & 1 & 1 \\
\hline 6 & N6 & 17 & 1 & -1 & P407 & -1 & -1 & 1 \\
\hline 7 & N7 & 11 & -1 & 1 & P407 & -1 & -1 & -1 \\
\hline 8 & N8 & 2 & 1 & 1 & P407 & -1 & 1 & -1 \\
\hline 9 & N9 & 12 & -1 & -1 & PVA & 1 & -1 & 1 \\
\hline 10 & N10 & 18 & 1 & -1 & PVA & 1 & 1 & 1 \\
\hline 11 & N11 & 5 & -1 & 1 & PVA & 1 & 1 & -1 \\
\hline 12 & N12 & 1 & 1 & 1 & PVA & 1 & -1 & -1 \\
\hline 13 & N13 & 8 & -1 & -1 & P407 & 1 & 1 & -1 \\
\hline 14 & N14 & 16 & 1 & -1 & P407 & 1 & -1 & -1 \\
\hline 15 & N15 & 13 & -1 & 1 & P407 & 1 & -1 & 1 \\
\hline 16 & N16 & 4 & 1 & 1 & P407 & 1 & 1 & 1 \\
\hline 17 & N17 & 9 & 0 & 0 & PVA & 0 & 0 & 0 \\
\hline 18 & N18 & 15 & 0 & 0 & PVA & 0 & 0 & 0 \\
\hline 19 & N19 & 6 & 0 & 0 & PVA & 0 & 0 & 0 \\
\hline
\end{tabular}

Note: Exp., Experiment; $\mathrm{X}_{1}, \mathrm{PCL}$ concentration $(\% \mathrm{w} / \mathrm{V}) ; \mathrm{X}_{2}$, stabilizer concentration $(\% \mathrm{w} / \mathrm{V})$;

$\mathrm{X}_{3}$, stabilizer type; $\mathrm{X}_{4}$, CURC:PCL ratio $(\mathrm{w} / \mathrm{w}) ; \mathrm{X}_{5}$, volume of acetone $(\mathrm{ml}) ; \mathrm{X}_{6}$, stirring speed $(\mathrm{rpm})$

ratio of 1:100. The analysis was carried out at $25^{\circ} \mathrm{C}$ using a $\mathrm{Ne}-\mathrm{He}$ laser at a scattering angle of $90^{\circ}$. The measurements were performed in triplicate and the obtained results were expressed as mean \pm standard deviation (SD).

\section{Zeta potential measurement}

The zeta potential of CURC-PCL NP was determined by laser doppler electrophoresis using a Zetasizer Nano ZS (Malvern Panalytical, Malvern, UK), after an appropriate 100 -fold dilution with distilled water. The zeta potential was calculated based on the electrophoretic mobility. Each sample was analyzed three times, and the results were expressed as mean \pm SD.

\section{Drug content and entrapment} efficiency

The concentration of entrapped CURC in the PCL nanoparticles was assessed by high performance liquid chromatography (HPLC) on an Agilent 1100 Series chromatographic instrument (Agilent Technologies, Inc., Santa Clara, CA, USA) equipped with an autosampler and a fluorescence detector. The samples were separated on a Zorbax SB-C18 column ( $3 \times 100 \mathrm{~mm}, 3.5 \mu \mathrm{m}$ particle size) from Phenomenex (Torrance, CA, USA) in isocratic conditions. The mobile phase consisted of a 40:60 mixture of acetonitrile and $0.2 \%$ formic acid at a flow rate of $1 \mathrm{ml} /$ $\min$ at $30^{\circ} \mathrm{C} .5 \mu \mathrm{L}$ of sample were 
injected in the chromatographic system by the autosampler, and CURC fluorescence detection was achieved using an excitation wavelength of $425 \mathrm{~nm}$ and an emission wavelength of $533 \mathrm{~nm}$. The entrapment efficiency (EE) was calculated with the following equation:

$\mathrm{EE}(\%)=$ (amount of CURC in the nanoparticles/total amount of CURC) $\times 100$

\section{Statistical analysis}

The results obtained for the 19 formulations were analyzed using the Modde 12.1 Software. Data were fitted to polynomial regression equations which indicated the effects of the investigated independent variables on the responses. The chosen model's fit was confirmed by the coefficient of determination $\left(R^{2}\right)$, the coefficient of prediction $\left(Q^{2}\right)$, the model validity and the reproducibility. Furthermore, the results were statistically validated by Analysis of Variance (ANOVA) for which a $p$ value lower than 0.05 was considered statistically significant. Similarly, the lack of fit was also calculated, and a $p$ value greater than 0.05 indicated insignificant model error.

\section{RESULTS AND DISCUSSIONS}

\section{Nanoparticle formulation and preparation method}

Nanoparticles, as drug delivery systems, have been developed to address the shortcomings of various drugs, including CURC, such as low water solubility $(1.34 \mathrm{\mu g} / \mathrm{ml}$ at $\left.25^{\circ} \mathrm{C}\right)$, chemical instability, and reduced bioavailability. The incorporation of CURC in polymeric nanoparticles offers several advantages, including increased physicochemical protection, controlled drug release and prolonged blood circulation [1]. PCL is an FDA-approved biodegradable semicrystalline polyester, widely used in the biomedical field for various medical devices like sutures, wound dressings, medical implants, even in tissue engineering, but mostly for drug delivery systems $[11,16]$. Because of its appealing properties, such as biodegradability, biocompatibility, high permeability to many drugs, $\mathrm{PCL}$ is a promising material for developing nano-drug delivery systems for safe and long-term use $[1,7]$. Therefore, CURC-PCL NP could be a suitable approach to reduce CURC limitations, and improve the therapeutic outcome.

A stabilizer or surfactant is typically used to stabilize the droplets of organic phase, and prevent aggregation. The type of surfactant is particularly important in order to obtain particles in the nanometer range, and with a narrow size distribution. Also, the biocompatibility and toxicity of the stabilizer must be taken into consideration in the selection process [21]. PVA and P407 were proposed as stabilizers in the present study due to their excellent biocompatibility $[22,23]$.

In the present study, the CURCloaded nanoparticles were obtained by the solvent displacement method also known as the nanoprecipitation method, widely employed for nanoparticle manufacturing. This technique is advantageous because it is simple, less expensive, requires less energy and materials, and few additives or even none at all. For this method, both the polymer and the drug must be soluble in the same organic solvent, but insoluble in a second solvent, typically water. 
Furthermore, the solvent and the non-solvent must be miscible [24]. In this study, we used acetone as the organic phase, since both CURC and PCL have good solubility in this solvent. Upon addition of the organic phase to the aqueous medium, a rapid desolvation of the polymer occurs, and due to the diffusion of the organic solvent in the dispersing medium, the polymer precipitates and entraps the drug inside the particles [24].

\section{Differential Scanning Calorimetry (DSC)}

DSC is extensively used in drug analysis to provide information about the physical and energetic properties of drugs [25]. Therefore, the compatibility of the materials used to prepare the CURC-PCL NP was investigated beforehand. The DSC thermograms of CURC, PCL, P407, PVA and the corresponding 1:1 (w/w) physical mixtures of CURC and each excipient are shown in Figure 1. The thermogram of CURC (Figure 1(a)) shows a sharp endothermic peak around $174.43^{\circ} \mathrm{C}$ and another smaller adjacent peak at $166.90^{\circ} \mathrm{C}$ which correspond to its melting. Other research groups reported a melting temperature for CURC of $178^{\circ} \mathrm{C}$ or $183^{\circ} \mathrm{C}[26,27]$, therefore our findings are similar to previously published data. PCL and P407 showed characteristic endothermic peaks at $58.86^{\circ} \mathrm{C}$ and $57.45^{\circ} \mathrm{C}$ (Figure 1 (b) and $1(\mathrm{c})$ ), respectively due to their melting. These results are in accordance with those published by others $[11,25]$. The DSC thermogram for PVA (Figure 1(d)) shows several endothermic peaks. The first one occurs at $55.12^{\circ} \mathrm{C}$ and corresponds to its glass transition temperature which is related to the amorphous part, while the next two peaks with maximum values at $165.27^{\circ} \mathrm{C}$ and $192.08^{\circ} \mathrm{C}$ probably refer to the melting of the crystalline part of the polymer. The last peak, over $250^{\circ} \mathrm{C}$, corresponds to the polymer's degradation. Our results are slightly different from those of Jamil et al. who reported for PVA a glass transition temperature of 20- $27^{\circ}$ and a melting temperature of $185-225^{\circ} \mathrm{C}$, depending on the degree of hydrolysis of the material [28]. The thermograms for the 1:1 $(w / w)$ physical mixtures (Figure $1(\mathrm{e})$-(g)) reveal the presence of all the endothermic peaks identified for the pure substances which means that the drugs were still in crystalline state. However, the endothermic peak for CURC shifted to lower melting points and decreased in intensity, probably due to the dilution effect of the polymers [25]. Our DSC results indicate that there was no visible interaction between CURC and either excipient.

\section{Nanoparticle characterization}

A total of 19 experiments were performed according to the fractional factorial design. The obtained nanoparticles were characterized in terms of size, PDI, zeta potential, CURC content and entrapment efficiency, and the results are presented in Table 3.

\section{Experimental data analysis. Model validation}

The experimental data were fitted to various models, and the results for the regression analysis are shown in Figure 2. The summary of fit plot showing the coefficient of determination $\left(R^{2}\right)$, the coefficient of prediction $\left(Q^{2}\right)$, the model validity and the reproducibility gives information on the adequacy and robustness of the model. $\mathrm{R}^{2}$, also termed "goodness of fit", had a score greater than 0.5 for all the responses, which indicated a good fit of the experimental data to the 
TABLE 3. Results for nanoparticle characterization

\begin{tabular}{|c|c|c|c|c|c|c|}
\hline No. & Exp. & $\begin{array}{c}\text { Size } \\
(\mathbf{n m})\end{array}$ & PDI & $\begin{array}{c}\text { Zeta } \\
\text { potential } \\
(\mathbf{m V})\end{array}$ & $\begin{array}{c}\text { CURC content } \\
(\mu \mathrm{g} / \mathrm{ml})\end{array}$ & $\begin{array}{c}\text { Entrapment } \\
\text { efficiency } \\
(\%)\end{array}$ \\
\hline 1 & N1 & $268.3 \pm 1.5$ & $0.033 \pm 0.023$ & $-15.8 \pm 0.9$ & $1454.4 \pm 48.5$ & $57.7 \pm 1.9$ \\
\hline 2 & N2 & $273.4 \pm 1.8$ & $0.052 \pm 0.036$ & $-20.3 \pm 0.9$ & $4777.5 \pm 97.3$ & $64.2 \pm 1.3$ \\
\hline 3 & N3 & $223.5 \pm 2.5$ & $0.043 \pm 0.037$ & $-13.9 \pm 1.4$ & $947.6 \pm 104.6$ & $38.4 \pm 4.2$ \\
\hline 4 & N4 & $353.7 \pm 5.6$ & $0.119 \pm 0.028$ & $-20.0 \pm 0.5$ & $1044.1 \pm 143.6$ & $13.9 \pm 1.9$ \\
\hline 5 & N5 & $248.5 \pm 2.2$ & $0.127 \pm 0.032$ & $-7.1 \pm 0.2$ & $1433.5 \pm 217.7$ & $58.3 \pm 8.8$ \\
\hline 6 & N6 & $295.1 \pm 5.5$ & $0.069 \pm 0.045$ & $-30.5 \pm 0.5$ & $737.3 \pm 468.0$ & $9.7 \pm 6.1$ \\
\hline 7 & N7 & $244.5 \pm 4.8$ & $0.056 \pm 0.032$ & $-27.5 \pm 2.3$ & $1783.7 \pm 30.9$ & $66.6 \pm 1.2$ \\
\hline 8 & N8 & $256.2 \pm 1.4$ & $0.042 \pm 0.030$ & $-33.1 \pm 0.6$ & $6277.4 \pm 20.7$ & $80.5 \pm 0.3$ \\
\hline 9 & N9 & $257.9 \pm 2.2$ & $0.085 \pm 0.033$ & $-8.8 \pm 0.9$ & $2811.2 \pm 43.1$ & $22.2 \pm 0.3$ \\
\hline 10 & N10 & $266.5 \pm 3.6$ & $0.052 \pm 0.049$ & $-6.3 \pm 0.2$ & $10524.2 \pm 3826.0$ & $27.8 \pm 10.1$ \\
\hline 11 & N11 & $256.9 \pm 4.4$ & $0.083 \pm 0.045$ & $-18.0 \pm 0.2$ & $7690.5 \pm 54.2$ & $61.9 \pm 0.4$ \\
\hline 12 & N12 & $326.8 \pm 7.0$ & $0.051 \pm 0.040$ & $-18.3 \pm 0.4$ & $19311.2 \pm 11141.8$ & $51.2 \pm 29.6$ \\
\hline 13 & N13 & $210.4 \pm 2.3$ & $0.09 \pm 0.054$ & $-27.5 \pm 2.3$ & $6597.7 \pm 100.8$ & $52.6 \pm 0.8$ \\
\hline 14 & N14 & $270.6 \pm 5.1$ & $0.109 \pm 0.056$ & $-33.6 \pm 0.5$ & $7111.5 \pm 33.3$ & $18.8 \pm 0.1$ \\
\hline 15 & N15 & $234.8 \pm 2.5$ & $0.134 \pm 0.026$ & $-9.7 \pm 1.2$ & $8303.0 \pm 2170.3$ & $66.5 \pm 17.4$ \\
\hline 16 & N16 & $236.8 \pm 3.1$ & $0.13 \pm 0.031$ & $-23.5 \pm 0.4$ & $16935.6 \pm 495.4$ & $44.8 \pm 1.3$ \\
\hline 17 & N17 & $269.2 \pm 2.2$ & $0.043 \pm 0.025$ & $-20.7 \pm 0.5$ & $11002.6 \pm 40.8$ & $65.6 \pm 0.2$ \\
\hline 18 & N18 & $255.2 \pm 3.2$ & $0.053 \pm 0.015$ & $-23.0 \pm 0.5$ & $7883.6 \pm 328.9$ & $47.0 \pm 2.0$ \\
\hline 19 & N19 & $264.4 \pm 1.4$ & $0.034 \pm 0.004$ & $-22.7 \pm 0.5$ & $11131.8 \pm 330.4$ & $66.6 \pm 2.0$ \\
\hline
\end{tabular}

Note: Exp., Experiment. Results are expressed as mean values \pm SD

generated model. For the particle size, size distribution and CURC content, $\mathrm{Q}^{2}$ or "goodness of prediction" was over the required value of 0.5 , indicating a strong predictive power of the model. However, for the zeta potential and the entrapment efficiency, the ability of the model to predict the effect of parameter variation on these responses was more reduced $\left(Q^{2}<0.5\right)$. The model validity and the reproducibility were over the ideal values of 0.25 and 0.5 , respectively, for all the observed responses, demonstrating a reduced pure error.

Additionally, the model was validated by performing the ANOVA test, and the results are presented in Table 4. For all the responses, the model was statistically significant $(\mathrm{p}<0.05)$, and with a low model error as demonstrated by the insignificant lack of fit values $(p>0.05)$. Overall, the ANOVA showed that the model was adequately chosen and valid.

The regression coefficients showing the effect of the studied independent variables on the observed responses are presented in Figure 3.

\section{Influence of variables on the nanoparticle size}

As indicated in Table 3, the size of the CURC-PCL NP varied between $210.4 \pm 2.3 \mathrm{~nm}$ and $353.7 \pm 5.6 \mathrm{~nm}$. According to Figure $3(a)$, the variables that had a significant effect $(p<0.05)$ on the particle size were the concentration of PCL $\left(X_{1}\right)$, the type of stabilizer $\left(X_{3}\right)$ and the volume of acetone $\left(X_{5}\right)$. The effects can be explained by the following regression equation:

$$
\begin{gathered}
Y_{1}=2.41+0.031 * X_{1}+0.020 * X_{3} \\
(P V A)-0.020 * X_{3}(P 407)-0.026 * X_{5}
\end{gathered}
$$


TABLE 4. Results for the ANOVA test for the observed responses

\begin{tabular}{|l|c|c|c|c|c|c|}
\hline \multicolumn{1}{|c|}{ Response } & DF & SS & $\begin{array}{c}\text { MS } \\
\text { (variance) }\end{array}$ & F & p & $\begin{array}{c}\text { Lack of } \\
\text { fit }\end{array}$ \\
\hline Size $(\mathrm{nm})$ & $15^{\mathrm{a}}$ & $0.012^{\mathrm{a}}$ & $0.0008^{\mathrm{a}}$ & $15.17^{\mathrm{b}}$ & 0.000 & 0.139 \\
\hline Polydispersity index & $13^{\mathrm{a}}$ & $0.005^{\mathrm{a}}$ & $0.0003^{\mathrm{a}}$ & $9.43^{\mathrm{b}}$ & 0.001 & 0.182 \\
\hline Zeta potential $(\mathrm{mV})$ & $15^{\mathrm{a}}$ & $491.11^{\mathrm{a}}$ & $32.74^{\mathrm{a}}$ & $7.84^{\mathrm{b}}$ & 0.002 & 0.071 \\
\hline CURC content $(\mu \mathrm{g} / \mathrm{ml})$ & $15^{\mathrm{a}}$ & $164811000^{\mathrm{a}}$ & $10987400^{\mathrm{a}}$ & $10.79^{\mathrm{b}}$ & 0.000 & 0.238 \\
\hline Entrapment efficiency $(\%)$ & $12^{\mathrm{a}}$ & $2001.57^{\mathrm{a}}$ & $166.79^{\mathrm{a}}$ & $6.45^{\mathrm{b}}$ & 0.004 & 0.478 \\
\hline
\end{tabular}

Note: ${ }^{a}$ Values for residuals; ${ }^{b}$ Values for regression

The strongest effect on particle size was observed for the polymer concentration $(p=0.0003)$. Increasing the PCL concentration led to an increase in particle size probably due to an increase in the viscosity of the organic solution, which consequently hampered the diffusion of the acetone in the aqueous phase and reduced the evaporation rate $[19,29]$.

Depending on the type of stabilizer, opposite effects $(p=0.009)$ were recorded on particle size. For PVA, a positive effect could be observed, which is in accordance with the results reported by Kasinathan et al. for CURC-loaded PLGA nanoparticles [30]. PVA acts as an emulsifying agent, reducing the interfacial tension between the organic and aqueous solutions, and typically favors the formation of smaller particles. Therefore, the effect observed for PVA could have been due to an insufficient amount of stabilizer, since PVA forms a continuous coat around the particles and opposes to the aggregation process [31]. However, at higher concentrations, the hydrophobic interactions between PVA molecules leads to aggregation and thus larger particles [29]. Additionally, some studies suggest that traces of PVA remain on the surface of the nanoparticles, even after repeated washing, which could also be responsible for the size increase [19]. In contrast, P407 contributed to the formation of smaller particles owing to its excellent stabilizing properties. In accordance with this, in a study conducted by Allotey-Babington et al., that investigated the stabilizing properties of three poloxamers (F-108, F-127 and P-188), the average size of dasatinib-loaded PCL nanoparticles prepared with F-127 (P407) was $184 \mathrm{~nm}$ [32]. A negative effect on particle size was also observed for the volume of organic solvent $(p=0.001)$. Increasing the volume of acetone favored a reduction in particle size, this effect being previously reported by our research group [20].

\section{Influence of variables on the polydispersity index (PDI)}

For the PDI, the values ranged from $0.033 \pm 0.023$ to $0.134 \pm 0.026$, which are indicative of a narrow distribution population of nanoparticles (Table 3).

Furthermore, the following regression equation was generated:

$$
\begin{gathered}
Y_{2}=0.071-0.006 * X_{1}-0.022 * X_{3} \\
(P V A)+0.022 * X_{3}(P 407)+0.016 * X_{4} \\
+0.009 * X_{6}
\end{gathered}
$$

As shown in Figure 3 (b), the type of stabilizer $\left(\mathrm{X}_{3}\right)$ and the CURC:PCL ratio $\left(X_{4}\right)$ had the strongest effect on the PDI ( $p=0.0004$ and $p=0.005$, respectively). PVA had a negative influence on the nanoparticle size distribution. Even though, as discussed above, PVA led to larger particles, it appeared that the formed nanoparticles were similar 
in size, and thus the PDI dropped. P407, however, had a contrary effect. Although the formulations which contained P407 showed lower particle size values, they proved to be less homogeneous in size. Colmenares Roldan et al. also found the surfactant to have an important effect on PDI, for PCL nanoparticles stabilized with Pluronic F-127 (P407) [33]. An increase in the PDI was also observed for higher CURC:PCL ratios. Our results are similar to those of Rafiei et al., who demonstrated that the polydispersity of PLGA nanoparticles increased with docetaxel concentration [34].

\section{Influence of variables on the zeta potential}

As shown in Table 3, the zeta potential values widely ranged from $-6.3 \pm 0.2 \mathrm{mV}$ to $-33.6 \pm 0.5 \mathrm{mV}$. PCL nanoparticles exhibited negative values for the zeta potential owing to the acidic $(\mathrm{COOH})$ groups of the polymer [35]. It is generally accepted that absolute zeta potential values greater that $60 \mathrm{mV}$ indicate great stability, $30 \mathrm{mV}$ values correspond to good stability, while values of $20 \mathrm{mV}$ indicate an acceptable short-term stability. Zeta potentials of less than $5 \mathrm{mV}$ are an indicator of the tendency of the nanoparticles to aggregate [35]. Therefore, in our study, the formulations N6, N8 and N14, for which zeta potential values of -30.5 $m V,-33.1 \mathrm{mV},-33.6 \mathrm{mV}$ have been obtained, could be considered stable, while the rest of the nanoparticles presented an acceptable or lower stability. However, this explanation is generally valid for low molecular weight stabilizers, but is not applicable for high molecular weight polymers that act by steric stabilization, like PVA. It is believed that PVA is adsorbed on the surface of the PCL nanoparticles and shields the superficial negative charges. In this case, even at low zeta potential values, the nanoparticle suspension is considered stable [35].

For the zeta potential, the regression equation was found to be:

$$
Y_{3}=-20.07-3.37 * X_{1}+3.49 * X_{3}
$$$$
\text { (PVA) }-3.49 * X_{3}(P 407)+4.37 * X_{6}
$$

The histograms in Figure 3 (c) depict a decrease in zeta potential with an increase in $\mathrm{PCL}$ concentration $\left(X_{1}, p=0.02\right)$ and with the use of P407 stabilizer $\left(X_{3}, p=0.02\right)$, since a larger number of $\mathrm{COOH}$ end groups would be available. For PVA $\left(\mathrm{X}_{3}\right)$, the positive effect on the zeta potential sustains the explanation given above. Rafiei et al. also found a positive correlation between the use of PVA as stabilizer and the surface charge of PLGA nanoparticles [34]. Moreover, in our study, the zeta potential increased with the stirring speed $\left(X_{6}, p=0.005\right)$, a finding also reported by Kasinathan et al. It is well known that a higher homogenization speed will lead to an increase of the total surface area of the nanoparticles, and consequently of the number of stabilizer-coated particles due to decrease in particle size [36].

\section{Influence of variables on CURC content}

The concentration of entrapped CURC in the polymeric nanoparticles ranged from $737.3 \pm 468.0 \mu \mathrm{g} / \mathrm{ml}$ to $19311.2 \pm 11141.8 \mu \mathrm{g} / \mathrm{ml}$ (Table 3). The regression equation that described the influence of the formulation factors on CURC content is given below:

$$
\begin{gathered}
Y_{4}=6724.12+2103.49 * X_{1}+ \\
1581.89 * X_{2}+3584.4 * X_{4}
\end{gathered}
$$


As shown in Figure $3(d)$, the CURC: $P C L$ ratio $\left(X_{4}\right)$ had a significant positive impact on the entrapped CURC content ( $p=0.0003)$. A larger amount of CURC in the organic phase implies a higher availability of the drug for incorporation in the PCL nanoparticles. At the same time, higher concentrations of CURC require a larger amount of hydrophobic polymeric matrix, which explains the positive impact of the PCL concentration $\left(X_{1}\right)$ on this response $(p=0.016)$. Similar results have been reported by Ravi et al. who developed PCL

nanoparticles loaded with lipophilic lopinavir [29].

\section{Influence of variables on the entrapment efficiency}

For the entrapment efficiency, values varied greatly from as low as $9.7 \pm 6.1 \%$ to as high as $80.5 \pm 0.3 \%$, as Table 3 indicates. The established polynomial equation for the entrapment efficiency was as follows:

$Y_{5}=60.50-4.67 * X_{1}+5.86 * X_{2}+$ $9.25 * X_{5}-12.23 * X_{6}-14.19 * X_{2}^{2}$
The variable with the highest magnitude of effect on the entrapment efficiency was the stirring speed $\left(X_{6}, p=0.002\right)$, followed by the volume of organic solvent $\left(X_{5}, p=0.012\right)$, as shown in Figure 3 (e). The homogenization speed had a negative effect on the entrapment efficiency. A more vigorous agitation of the nanoparticle suspension led to a decrease in the percentage of entrapped CURC.

On the other hand, increasing the volume of acetone contributed to an increase in the entrapment efficiency values. This is possibly related to the good solubility that CURC displays in acetone, which favors the interactions between the drug molecules and PCL. A similar observation was made by Ramalho et al. for temozolomide-loaded PLGA nanoparticles [19]. Rafiei et al. also found a decrease in drug loading efficiency of docetaxel in PLGA nanoparticles with the decrease in the organic/aqueous phase ratio. This trend was

\section{REFERENCES}

1. Umerska A, Gaucher C, Oyarzun-Ampuero $F$ et al. Polymeric Nanoparticles for Increasing Oral Bioavailability of Curcumin. Antioxidants (Basel) [Internet]. $2018 \mathrm{Apr}$ [cited 2020 Sep 25];7(4):46. Available from: https://www.ncbi.nlm.nih.gov/pmc/ articles/PMC5946112/.

2. Sun J, Chen F, Braun C et al. Role of curcumin in the management of pathological pain. Phytomedicine. 2018; 48:129-40

3. Naksuriya O, Okonogi S, Schiffelers RM et al. Curcumin nanoformulations: A review of pharmaceutical properties and preclinical studies and clinical data related to cancer treatment. Biomaterials. 2014; 35(10):3365-83

4. Del Prado-Audelo ML, Magaña JJ, Mejía-Contreras BA et al. In vitro cell uptake evaluation of curcumin-loaded PCL/ F68 nanoparticles for potential application in neuronal diseases. J Drug Deliv Sci Technol. 2019;52:905-14.

5. Youssouf L, Bhaw-Luximon $A$, Diotel $\mathrm{N}$ et al. Enhanced effects of curcumin encapsulated in polycaprolactone-grafted oligocarrageenan nanomicelles, a novel nanoparticle drug delivery system. Carbohydr Polym. 2019;217:35-45.

6. Curcuma longa [Internet]. Amsterdam (Netherlands): European Medicines Agency; [2019 Jan 24]. Assessment report on Curcuma longa L., rhizoma; [cited 2020 Sep 26]. Available from: https://www.ema. europa.eu/en/documents/herbal-report/ final-assessment-report-curcuma-longa-lrhizoma-revision-1_en.pdf. attributed to the leak of drug molecules into the aqueous medium [34].

\section{CONCLUSIONS}

As drug delivery systems, $\mathrm{PCL}$ nanoparticles have received a lot of attention due to their favorable characteristics such as biodegradability, biocompatibility, ease of manufacture, stability and possibility of controlled release. In the present study, PCL nanoparticles loaded with CURC, a natural polyphenol with pleiotropic effects, were prepared by the solvent displacement method. A fractioned factorial design helped identify the input variables with the highest impact on the CURC-PCL NP properties, and create a robust model with good predicting ability. This work emphasizes the usefulness of using the DOE approach in the development and manufacturing of CURC-PCL NP.

Conflict of interest: none declared Financial support: none declared
7. Bakre LG, Sarvaiya JI, Agrawal YK. Synthesis, Characterization, and Study of Drug Release Properties of Curcumin from Polycaprolactone /Organomodified Montmorillonite Nanocomposite. J Pharm Innov. 2016;11:300-7.

8. El-Say KM, El-Sawy HS. Polymeric nanoparticles: Promising platform for drug delivery. Int J Pharm. 2017;528(1-2):675-91.

9. Karlsson J, Vaughan HJ, Green JJ. Biodegradable Polymeric Nanoparticles for Therapeutic Cancer Treatments. Annu Rev Chem Biomol Eng. 2018;9:105-27.

10. Nagy NZ, Varga Z, Mihály J et al. Highly efficient encapsulation of curcumin into and $\mathrm{pH}$-controlled drug release from poly( $\varepsilon$-caprolactone) nanoparticles stabilized with a novel amphiphilic 
hyperbranched polyglycerol. Express Polym Lett. 2020;14(1):90-101.

11. Mohamed RM, Yusoh K. A Review on the Recent Research of Polycaprolactone (PCL). Adv Mat Res. 2015;1134:249-55.

12. Bartnikowski M, Dargaville TR, Ivanovski S et al. Degradation mechanisms of polycaprolactone in the context of chemistry, geometry and environment. Prog Polym Sci. 2019;96:1-20.

13. Witt S, Scheper T, Walter JG. Production of polycaprolactone nanoparticles with hydrodynamic diameters below $100 \mathrm{~nm}$. Eng Life Sci. 2019;19:658-65.

14. Mehrotra A, Pandit JK. Preparation and Characterization and Biodistribution Studies of Lomustine Loaded PLGA Nanoparticles by Interfacial Deposition Method. J Nanomed Nanotechnol [Internet]. 2015 Jan [cited 2020 Sep 25];6:6. Available from: https:// www.longdom.org/open-access/ preparation-and-characterization-andbiodistribution-studies-of-lomustineloaded-plga-nanoparticles-by-interfacialdeposition-method-2157-7439-1000328. pdf.

15. Piñón-Segundo E, Ganem-Quintanar A, Garibay-Bermúdez JR et al. Preparation of Nanoparticles by Solvent Displacement Using a Novel Recirculation System. Pharm Dev Technol. 2006;11(4):493-501.

16. Elmowafy M, Alruwaili NK, Shalaby K et al. Long-Acting Paliperidone Parenteral Formulations Based on Polycaprolactone Nanoparticles; The Influence of Stabilizer and Chitosan on In Vitro Release, Protein Adsorption, and Cytotoxicity. Pharmaceutics [Internet]. 2020 Feb [cited 2020 Sep 25];12(2):160. Available from: https://www. ncbi.nlm.nih.gov/pmc/articles/ PMC7076490/

17. Yuangyai C, Nembhard HB. Design of Experiments: A Key to Innovation in Nanotechnology. In: Ahmed W, Jackson MJ (eds.). Emerging Nanotechnologies for Manufacturing. $1^{\text {st }}$ ed. [place unknown]: William Andrew; 2009:207-34.

18. El-Ahmady El-Naggar N, Abelwahed NAM. Application of Statistical Experimental Design for Optimization of Silver Nanoparticles Biosynthesis by a Nanofactory Streptomyces viridochromogenes. J Microbiol. 2014; 52(1):53-63.

19. Ramalho MJ, Loureiro JA, Coelho MAN et al. Factorial Design as a Tool for the
Optimization of PLGA Nanoparticles for the Co-Delivery of Temozolomide and O6-Benzylguanine. Pharmaceutics [Internet]. 2019 Aug [cited 2020 Sep 25];11(8):401. Available from: https://www. ncbi.nlm.nih.gov/pmc/articles/ PMC6722980/

20. Tefas LR, Rus LM, Achim M et al. Application of the Quality by Design concept in the development of quercetin-loaded polymeric nanoparticles. Farmacia. 2018 ; 66(5):798-810.

21. Sharma N, Madan P, Lin S. Effect of process and formulation variables on the preparation of parenteral paclitaxel-loaded biodegradable polymeric nanoparticles: A co-surfactant study. Asian J Pharm Sci. 2016;11:404-16.

22. El Fawal G, Hong H, Song $X$ et al. Polyvinyl Alcohol/Hydroxyethylcellulose Containing Ethosomes as a Scaffold for Transdermal Drug Delivery Applications. Appl Biochem Biotechnol. 2020;191(4):1624-37.

23. Yu S, Zhang X, Tan $\mathrm{G}$. A novel $\mathrm{pH}$-induced thermosensitive hydrogel composed of carboxymethyl chitosan and poloxamer cross-linked by glutaraldehyde for ophthalmic drug delivery. Carbohydr Polym. 2017;155:208-17.

24. Salatin S, Barar J, Barzegar-Jalali M et al. Development of a nanoprecipitation method for the entrapment of a very water soluble drug into Eudragit RL nanoparticles. Res Pharm Sci. 2017;12(1):1-14.

25. Ei-Badry M, Hassan MA, Ibrahim MA et al. Performance of Poloxamer 407 as hydrophilic carrier on the binary mixtures with nimesulide. Farmacia. 2013; 61(6):1137-50.

26. Jahagirdar PS, Gupta PK, Kulkarni SP et al. Polymeric curcumin nanoparticles by a facile in situ method for macrophage targeted delivery. Bioeng Trans/ Med. 2019;4(1):141-51.

27. Dewangan AK, Varkey S, Mazumder S. Synthesis of Curcumin Loaded CMCAB Nanoparticles for Treatment of Rheumatoid Arthritis. In: Ahmadi R, Gaidamashvili M, editors. International Conference on Chemical, Environmental and Biological Sciences (CEBS-2015); 2015 Mar 18-19; Dubai, UAE. p. 25-30.

28. Jamil N, Husin H, Alfida AW et al. Characterization and Preparation of
Polyvinyl Alcohol (PVA) as Inhibitor in Formation of Hydrate. Int J Cur Res Eng Sci Tech. 2018;1(S1):578-84.

29. Ravi PR, Vats R, Dalal V et al. Design, optimization and evaluation of poly-ecaprolactone (PCL) based polymeric nanoparticles for oral delivery of lopinavir. Drug Dev Ind Pharm. 2015;41(1):131-40.

30. Kasinathan N, Amirthalingam M, Reddy ND et al. Polycaprolactone-based in situ implant containing curcumin-PLGA nanoparticles prepared using the multivariate technique. Artif Cells Nanomed Biotechnol. 2016; 44(6):1520-8.

31. Ribeiro AF, de Oliveira Rezende RL, Mendes Cabral L et al. Poly $\varepsilon$-caprolactone nanoparticles loaded with Uncaria tomentosa extract: preparation, characterization, and optimization using the Box-Behnken design. Int J Nanomedicine. 2013;8:431-42.

32. Allotey-Babington GL, Nettey H, D'Sa S et al. Cancer chemotherapy: Effect of poloxamer modified nanoparticles on cellular function. J Drug Deliv Sci Technol. 2018;47:181-92.

33. Colmenares Roldán GJ, Agudelo Gomez LM, Cornelio JAC et al. Production of polycaprolactone nanoparticles with low polydispersity index in a tubular recirculating system by using a multifactorial design of experiments. J Nanopart Res [Internet]. 2018 Mar 9 [cited 2020 Sep 26];20:68. Available from: https:// link.springer.com/article/10.1007/ s11051-018-4168-8.

34. Rafiei P, Haddadi A. A robust systematic design: Optimization and preparation of polymeric nanoparticles of PLGA for docetaxel intravenous delivery. Mater Sci Eng C [Internet]. 2019 Jul [cited 2020 Sep 26];104:109950. Available from: https:// www.sciencedirect.com/science/article/ abs/pii/S0928493118334490.

35. Miladi K, Sfar S, Fessi H et al. Encapsulation of alendronate sodium by nanoprecipitation and double emulsion: From preparation to in vitro studies. Ind Crops Prod. 2015; 72:24-33.

36. Kasinathan N, Amirthalingam M, Reddy ND et al. In-situ implant containing PCLcurcumin nanoparticles developed using design of experiments. Drug Deliv. 2016; 23(3)1007-15 